\title{
Lost time: Bindings do not represent temporal order information
}

\author{
Birte Moeller ${ }^{1} \cdot$ Christian Frings $^{1}$ \\ Published online: 4 June 2018 \\ (C) Psychonomic Society, Inc. 2018
}

\begin{abstract}
Many accounts of human action control assume bindings between features of stimuli and responses of individual events. One widely accepted assumption about these bindings is that they do not contain temporal-order representations regarding the integrated elements. Even though several theories either explicitly or implicitly include it, this assumption has never been tested directly. One reason for this lack of evidence is likely that typical stimulusresponse binding paradigms are inapt for such a test. Adapting a new paradigm of response-response binding to include order switches between response integration and retrieval, we were able to analyze possible representation of order information in bindings for the first time. Binding effects were identical for intact and switched response orders, indicating that bindings indeed include no temporal-order information.
\end{abstract}

Keywords Action control $\cdot$ SR-binding $\cdot$ Binary binding $\cdot$ Temporal order

Many everyday actions, like reaching for your cup of coffee, seem to be executed easily and smoothly_-most humans likely do not even think about these seemingly simple actions. Yet theories on action control suggest that simple actions are anything but simple. In fact, it is assumed that stimuli and actions are represented in feature codes and that these feature codes are bound together to produce action plans (Henson, Eckstein, Waszak, Frings, \& Horner, 2014; Hommel, 2004). Such action plans dissolve over time after an action has been executed, but clearly they do not so immediately. So, if a feature from such an action is repeated, typically the whole previous episode is retrieved and can then influence the current action. For this reason binding between feature codes that occurred in event $n-1$ can be measured via retrieval due to feature repetition in event $n$. This logic has been applied to a wide array of action control tasks, like negative priming (for a review, see Frings, Schneider, \& Fox, 2015), task switching (for a review, Kiesel et al., 2010), the Gratton effect

Birte Moeller

moellerb@uni-trier.de

1 Cognitive Psychology, Department of Psychology, University of Trier, Campus I, 54286 Trier, Germany
(Gratton, Coles, \& Donchin, 1992), or repetition priming (Henson et al., 2014). ${ }^{1}$ Obviously, stimuli and actions of an individual event can arguably be distributed over time. Yet various retrieval theories (e.g., Hommel, Müsseler, Aschersleben, \& Prinz, 2001; Kunde, Kiesel, \& Hoffmann, 2003; Logan, 1988, 1990; Schmidt, De Houwer, \& Rothermund, 2016) that explain the abovementioned effects in terms of binding and retrieval of feature codes either implicitly or explicitly make the assumption that (ordinal) temporal order information is not part of bindings in action control.

The instance theory of automatization (Logan, 1988, 1990) assumes that each instance of an event is stored in long-term memory and that attention to a repeated stimulus starts retrieval of stored instances. Yet it does not account for instances that are distributed over time. An instance appears to be the snapshot of a single moment, accounting for stimulus-response ( $\mathrm{S}-\mathrm{R}$ ) bindings, and by accumulating instances, also for automatization learning. The parallel episodic processing model (Schmidt et al., 2016) is a computational model accounting for various well-known effects in action control via an accumulation of instances

\footnotetext{
${ }^{1}$ Even though bindings can influence subsequent performance for a short time after integration, they can be assumed to be distinct from SR-learning, which influences action control on a longer term basis (e.g., Herwig \& Waszak, 2012; Moeller \& Frings, 2017).
} 
similar to those assumed by Logan. It does make the assumption that response timing is represented in each stored episode. Yet temporal order information regarding individual parts of the episode are not defined as part of an episode representation. Finally, the theory of event coding (TEC; Hommel et al., 2001) explicitly points out that the binary quality of bindings between features within an event file implies (1) that bindings always involve two features of an event and, more importantly in this context, (2) that repetition of one can retrieve the other, independent of their original order when first experienced (Hommel, 2009).

Despite such wide consensus regarding its accuracy, the assumption that temporal-order information is not represented in bindings was never explicitly tested. In fact, results in related fields provide reason to doubt its accuracy by suggesting that order of stimulus occurrence can easily be both perceived and also recalled: Accurate temporal order judgements in perception research indicate that even relatively small differences of $40 \mathrm{~ms}$ interstimulus intervals are encoded (e.g., Kanabus, Szelag, Rojek, \& Pöppel, 2002). In addition, recall of perceived temporal order from working memory has been used widely to study working memory characteristics (e.g., Baddeley, 1966; Henson, Norris, Page, \& Baddeley, 1996).

Binding has typically been shown with feature compounds that were presented simultaneously and thus did not contain episodic order information (e.g., Frings \& Rothermund, 2011; Giesen \& Rothermund, 2011; Hommel, 1998; Mayr \& Buchner, 2006). One exception are studies regarding response effect (RE) binding, which indeed analyze integration of sequentially occurring events (e.g., Dutzi \& Hommel, 2009; Herwig \& Waszak, 2012; Janczyk, Heinemann, \& Pfister, 2012; Moeller, Pfister, Kunde, \& Frings, 2016). Nevertheless, these studies cannot give insight into representation of order information, either. Bindings between a response and its following effect are measured in the subsequent event. Shortly after a response triggered an effect, this same effect or a different stimulus is presented. Participants' task is to execute a free response at this stimulus presentation. More repetitions of former responses, if the effect is repeated than if a different stimulus is presented, is interpreted as indicating short-term binding between response and effect identity. Yet this result does not imply anything about a possible representation of temporal-order information: Binding between response and effect identity can be assumed to be independent of other representations within the event file (e.g., Giesen \& Rothermund, 2014; Hommel, 1998; Moeller, Frings, \& Pfister, 2016). To test for representation of order information, retrieval effects in the typical RE-binding paradigm would need to be compared with retrieval effects, measured in an intact temporal order (response execution $\rightarrow$ effect presentation). Notably, it is impossible to decide whether retrieval would be stronger for intact RE order because response retrieval (measured in the typical paradigm) and stimulus feature retrieval (e.g., Müsseler \& Hommel, 1997) cannot be measured via the same dependent variable. Both retrieval effects are thus difficult to compare.

However, the TEC is grounded on the assumption that there is essentially no difference between stimulus and response representations (Hommel et al., 2001), and it has recently been shown that binding between two subsequent and individually planned responses behaves essentially like $\mathrm{S}-\mathrm{R}$ binding (R-R binding; Moeller \& Frings, manuscript submitted for publication). In a prime-probe design including two responses (R1 and R2) in each prime and each probe, we varied response relations (repetition vs. change) orthogonally between prime and probe. Binding took place between the two prime responses, and, subsequently, repeating prime R1 as probe $\mathrm{R} 1$ retrieved prime $\mathrm{R} 2$. This retrieval led to response facilitation if the same response had to be repeated as probe $\mathrm{R} 2$, and to impairment if a different response was required. Thus, R-R-binding effects are indicated by an interaction between response R1 relation (repetition vs. change from prime to probe) and response $\mathrm{R} 2$ relation (repetition vs. change). With this finding it becomes possible to directly investigate whether or not temporal order information can be represented in bindings (for a visualization of predictions regarding the two possible representations within an event file, see Fig. 1a-b). Slightly adapting the paradigm, here we allowed switches of response order between integration and retrieval. If response order is indeed not represented in bindings, binding effects should be identical regardless whether the response starting retrieval (probe R1) was previously given as prime R1 or prime R2. However, if bindings include response order representations, binding effects for changed response order should be significantly smaller than for intact response order.

\section{Experiment}

\section{Method}

Participants Based on past experiments, we expected at least a medium-sized effect. With $\alpha=.05,1-\beta=.9$, and a correlation among repeated measures of at least $.5,30$ participants were necessary (Faul, Erdfelder, Lang, \& Buchner, 2007). Thirty students ( 25 female) from the University of Trier took part in the experiment. The median age of the sample was 22 years (range: 19-35). All participants reported normal or corrected-to-normal vision and received partial course credit.

Design The design comprised three within-subjects factors: Response R1 relation (response repetition vs. response change from prime to probe), response $\mathrm{R} 2$ relation (response repetition vs. response change from prime to probe), and order of $\mathrm{R} 1$ and $\mathrm{R} 2$ in prime and probe (same vs. different). 
Event File with

temporal order information
Event File without

temporal order information

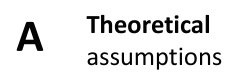

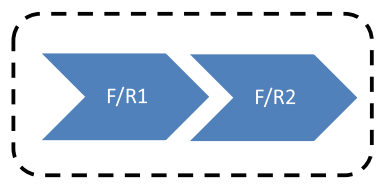

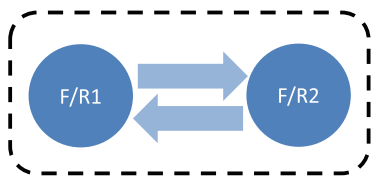

(i) Repetition of $F / R 1$ retrieves $F / R$ encoded after $F / R 1$ ( $=F / R 2$ )

(ii) Repetition of $F / R 2$ retrieves $F / R$ encoded before F/R2 (=F/R1)

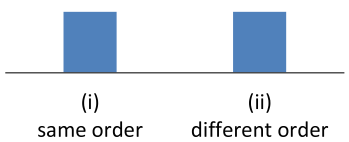

\section{Observed data}

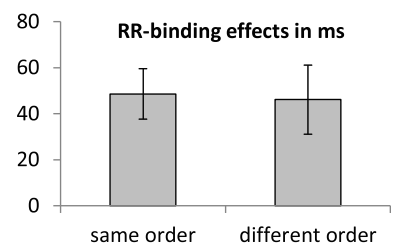

(ii) Repetition of F/R2 does not retrieve $F / R$ encoded before F/R2 (=F/R1)

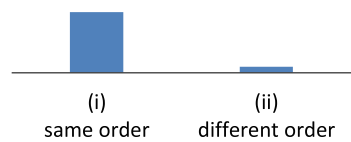

Fig. 1 a Visualization of theoretical assumptions for the case that temporal order information becomes part of bindings (left-hand side) and that temporal order information does not become part of bindings. b Predictions of binding effects regarding the two possibilities: Same order indicates that any repeated response in the probe sequence remained at its position from the prime sequence (i.e., prime $\mathrm{R} 1$ repeated in the probe, could retrieve prime $\mathrm{R} 2$ ); different order indicates that any repeated response in the probe sequence had a different temporal position as

Materials The experiment was conducted using E-Prime 2.0. Instructions and stimuli were shown in white on black background on a standard liquid crystal display (TFT) screen. Stimuli were the letters A, B, C, and D and the digits 1, 2, 3, and 4 . All letters and digits subtended a horizontal visual angle of $0.4^{\circ}$ to $0.6^{\circ}$, and vertical visual angle of $0.5^{\circ}$. Viewing distance was approximately $60 \mathrm{~cm}$. Participants responded by pressing one of four keys on the computer keyboard.

Procedure Participants were tested individually in soundproof chambers. Instructions were given on the screen. Participants placed middle and index fingers on the keys S, C, M, and L of a standard computer keyboard, which were marked with $\mathrm{A} / 1$, $\mathrm{B} / 2, \mathrm{C} / 3$, and $\mathrm{D} / 4$. Their task was always to press the key corresponding to the individually presented letters and digits. All stimuli were presented centered on the screen. The beginning of each trial (see Fig. 2) was indicated by an asterisk, presented for 1,000 ms. It was exchanged for a plus sign for $500 \mathrm{~ms}$, which was followed by the first prime stimulus (letter or digit) indicating prime response $\mathrm{R} 1$ until response execution. Then the second prime stimulus appeared indicating prime response $\mathrm{R} 2$ until response execution. Then a fixation mark appeared for $500 \mathrm{~ms}$ and was followed by the first probe stimulus indicating probe R1 until response execution. Then the second probe stimulus appeared indicating probe R2 until response execution. In case of an incorrect response R1 or R2 in prime or probe, an error message appeared for $1,500 \mathrm{~ms}$ immediately following the erroneous response, reminding the participant to respond as quickly as possible, but without making errors. Every 40 trials participants were allowed to take a short break, after which they resumed the task in their own time. compared with the prime sequence (i.e., prime $\mathrm{R} 2$, repeated in the probe, could retrieve prime R1). c Binding effects, observed in the present study, for trials with same (left-hand side) and different (right-hand side) response order from prime to probe sequence. Binding effects are calculated as $\mathrm{R} 1$ repetition minus R1 change RTs for R2 change trials, subtracted from $\mathrm{R} 1$ repetition minus $\mathrm{R} 1$ change $\mathrm{RTs}$ for $\mathrm{R} 2$ repetition trials ((R1cR2r $-\mathrm{R} 1 \mathrm{rR} 2 \mathrm{r})-(\mathrm{R} 1 \mathrm{cR} 2 \mathrm{c}-\mathrm{R} 1 \mathrm{rR} 2 \mathrm{c}))$

The relation of $\mathrm{R} 1$ between prime and probe (repetition vs. change) was varied orthogonally to the relation of R2 (repetition vs. change). For example trials of all eight conditions, see Table 1. In R1 repetition trials (R1r), the same response was required to the stimulus indicating prime response $\mathrm{R} 1$ and the one indicating probe response R1. In R1 change trials (R1c), different responses were required to the stimulus indicating prime response $\mathrm{R} 1$ and the one indicating probe response $\mathrm{R} 1$. In $\mathrm{R} 2$ repetition trials ( $\mathrm{R} 2 \mathrm{r}$ ), the same response was required to the stimulus indicating prime response $\mathrm{R} 2$ and the one indicating probe response R2. In R2 change trials (R2c), different responses were required to the stimulus indicating prime response $\mathrm{R} 2$ and the one indicating probe response $\mathrm{R} 2$. These relations resulted in the four conditions $\mathrm{R} 1 \mathrm{rR} 2 \mathrm{r}$, R1rR2c, R1cR2r, and R1cR2c. All four conditions could be presented with the same response order in prime and probe and with a different response order, which resulted in eight conditions. In the different-order conditions, R1 relation referred to that between probe response $\mathrm{R} 1$ and prime response $\mathrm{R} 2$ and $\mathrm{R} 2$ relation referred to the relation between probe response $\mathrm{R} 2$ and prime response $\mathrm{R} 1$. Thus, for example, an R1rR2c trial with a different response order could be prompted by the prime stimuli $\mathrm{B} \rightarrow 1$ and the probe stimuli $A \rightarrow 3$. Each of the eight conditions was presented eight times with each of the four possible combinations of letter and digit (letter/letter, letter/digit, digit/letter, digit/digit) indicating probe responses R1 and R2, resulting in 256 experimental trials. Stimuli were randomly assigned to indicate R1 and R2 in prime and probe, with the restrictions due to the requirements of the current condition and the restriction that none of the stimuli was repeatedly presented in one trial. For example, 


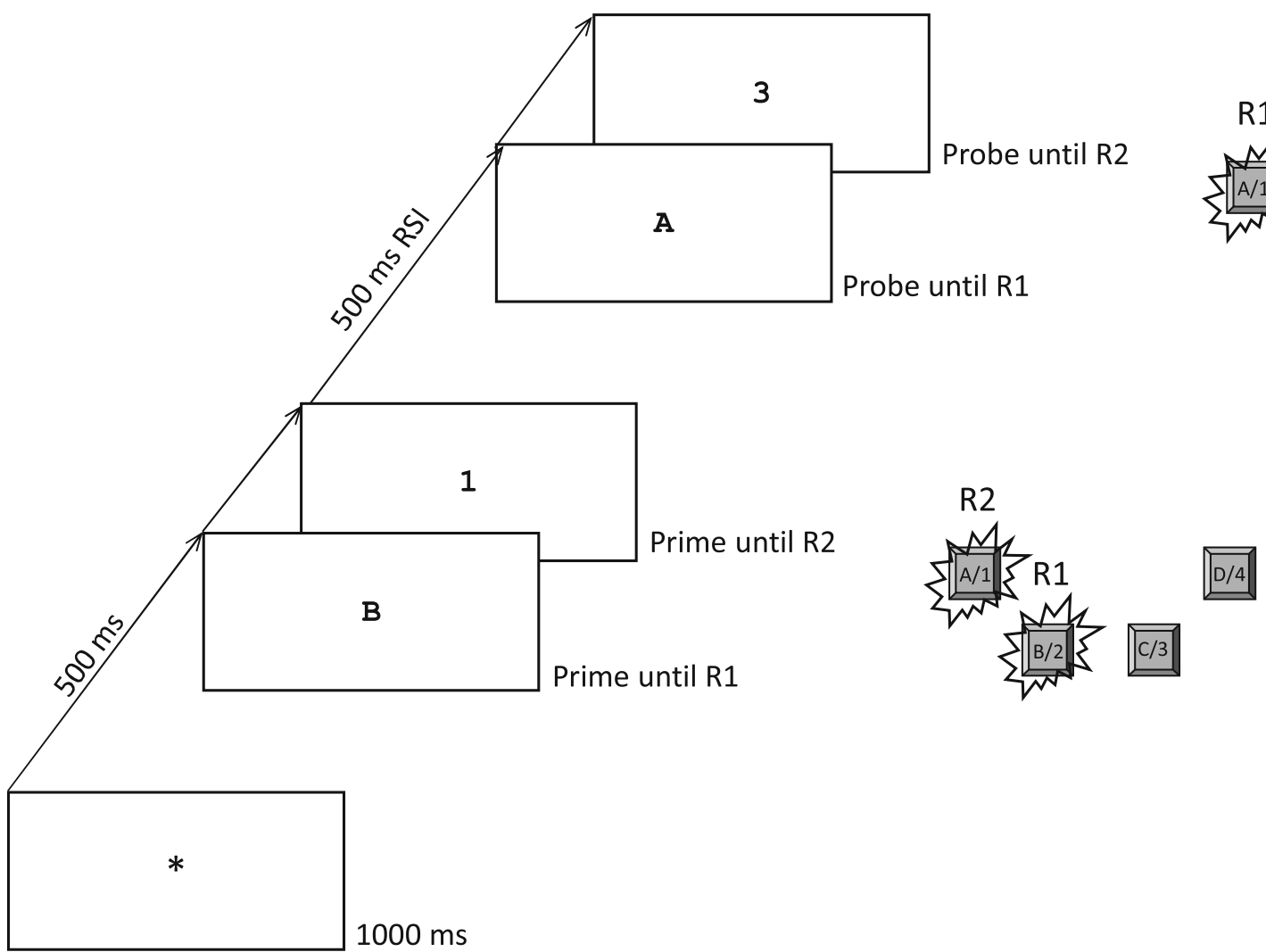

Fig. 2 Schematic example for the sequence of events in one trial. Participants gave two successive responses, R1 and R2, both to the prime and to the probe. This is an example for a R1 repetition and R2

if prime responses R1 and R2 were indicated by 1 and 3 in a $\mathrm{R} 1 \mathrm{rR} 2 \mathrm{r}$ trial, probe responses would be indicated by A and C. Before the experimental block started, participants practiced their task for 32 trials (subsample of the experimental trials).

\section{Results}

Our dependent variable of interest was performance in probe $\mathrm{R} 2$ : If prime R1 and prime R2 were integrated, repeating one of them as probe R1 may trigger retrieval of the other, influencing probe $\mathrm{R} 2$ performance. For the analysis of change trial with different response orders in prime and probe. White is depicted in black and black is depicted in white; stimuli are not drawn to scale

response times (RTs), we considered only those trials with correct responses R1 and R2 both in prime and probe. The rate for at least one prime response error was $5.1 \%$. Probe error rates were $2.5 \%$ for R1, and $2.6 \%$ for R2 (only including trials with correct previous responses). RTs that were more than 1.5 interquartile ranges above the third quartile of the RT distribution of the participant (Tukey, 1977), and RTs that were shorter than $200 \mathrm{~ms}$ were excluded from the analysis. Due to these constraints, $13.8 \%$ were excluded from the RT analyses. For mean RTs and error rates, see Table 2.

In a 2 (R1 relation: repetition vs. change) $\times 2$ ( 2 relation: repetition vs. change) $\times 2$ (response order: same vs. different)

Table 1 Examples for stimuli presented to indicate prime and probe responses R1 and R2 for all eight conditions

\begin{tabular}{|c|c|c|c|c|c|}
\hline Order & $\mathrm{R} 1$ relation & $\mathrm{R} 2$ relation & & Prime stimuli & Probe stimuli \\
\hline \multirow[t]{4}{*}{ Same } & \multirow[t]{2}{*}{ Repetition } & Repetition & (R1rR2r same) & $4 \rightarrow \mathrm{C}$ & $\mathrm{D} \rightarrow 3$ \\
\hline & & Change & (R1rR2c same) & $4 \rightarrow \mathrm{B}$ & $\mathrm{D} \rightarrow 3$ \\
\hline & \multirow[t]{2}{*}{ Change } & Repetition & (R1cR2r same) & $A \rightarrow C$ & $\mathrm{D} \rightarrow 3$ \\
\hline & & Change & (R1cR2c same) & $A \rightarrow B$ & $\mathrm{D} \rightarrow 3$ \\
\hline \multirow[t]{4}{*}{ Different } & \multirow[t]{2}{*}{ Repetition } & Repetition & (R1rR2r different) & $\mathrm{C} \rightarrow 4$ & $\mathrm{D} \rightarrow 3$ \\
\hline & & Change & (R1rR2c different) & $\mathrm{B} \rightarrow 4$ & $\mathrm{D} \rightarrow 3$ \\
\hline & \multirow[t]{2}{*}{ Change } & Repetition & (R1cR2r different) & $\mathrm{C} \rightarrow \mathrm{A}$ & $\mathrm{D} \rightarrow 3$ \\
\hline & & Change & (R1cR2c different) & $\mathrm{B} \rightarrow \mathrm{A}$ & $\mathrm{D} \rightarrow 3$ \\
\hline
\end{tabular}


Table 2 Mean response times (in $\mathrm{ms}$ ) and mean error rates (in percentage) for probe responses R1 and R2, as a function of response order as well as R1 relation and R2 relation between prime and probe

Same order

$\mathrm{R} 2$ repetition $\mathrm{R} 2$ change $\mathrm{R} 2$ repetition $\mathrm{R} 2$ change

Probe R2

R1 change

R1 repetition

$630(2.7)$

$599(2.2)$

$633(3.2)$

$598(1.4)$

Priming effect

$601(2.7)$

$619(2.9)$

$600(2.9)$

610 (2.9)

$29(0.0)$

$-20(-0.7)$

$33(0.3)$

$-12(-1.5)$

MANOVA on probe response R2 RTs with Pillai's trace as the criterion, the main effect of $\mathrm{R} 2$ relation was significant, $F(1$, 29) $=12.90, p=.001 \eta_{\mathrm{p}}{ }^{2}=.31$. Participants responded faster if R2 was changed $(M=606 \mathrm{~ms}, S D=106)$ than if it had to be repeated $(M=616 \mathrm{~ms}, S D=105)$ from prime to probe. More importantly, the interaction of R1 and R2 relation was significant as well, $F(1,29)=34.60, p<.001, \eta_{\mathrm{p}}{ }^{2}=.54$, indicating binding between the responses. Most importantly, this binding effect was not further modulated by response order, $F(1,29)<$ $1, p=.907 \eta_{\mathrm{p}}{ }^{2}<.01$ (see Fig. 1c). A power analysis revealed a power of $1-\beta=.92$ for finding even a small difference between binding effects (Faul et al., 2007), and a Bayes factor of $\mathrm{BF}_{01}=5.11$ (calculated via JASP) indicated that the data are 5.11 times more likely under the null hypothesis that postulates identical binding effects than under the alternative hypothesis that postulates a difference between binding effects (Wagenmakers et al., 2017). In addition, the interaction of R1 relation and response order approached significance, $F(1,29)$ $=3.53, p=.070, \eta_{\mathrm{p}}{ }^{2}=.11$. None of the other effects was significant, $F_{\mathrm{s}}<2.6, p \mathrm{~s}>.1, \eta_{\mathrm{p}}{ }^{2} \mathrm{~s}<.09$. In the same analyses on error rates, the numerical pattern was similar, but none of the effects reached significance, $F<2.7, p>.1, \eta_{\mathrm{p}}{ }^{2}<.09$.

\section{Discussion}

The present study investigated whether or not order information is represented as part of a binding between two subsequently executed responses. Each prime and each probe consisted of two subsequent responses (R1 and R2). The significant interaction of $\mathrm{R} 1$ relation and $\mathrm{R} 2$ relation together with the nonsignificant three-way interaction indicate that independent of the former response order, repeating either of the responses from the prime as the first response in the probe, triggered retrieval of the other response. The result pattern suggests that bindings between individual elements of events do not include temporal order representations.

Our result is in line with a number of current retrieval theories that implicitly or explicitly assume a lack of order information within one instance or event file (e.g., Hommel et al., 2001; Logan, 1988; Schmidt et al., 2016). We were able to provide for the first time unambiguous evidence for this central assumption. Note that order information in SR compounds, especially regarding response irrelevant stimuli, are hardly ever salient (e.g., Hommel, 2005). In contrast, here participants triggered the progress of stimulus presentation by responding to individually presented stimuli. Intervals between responses extended about 600-700 ms. Thus, we can assume that stimulus and response order was encoded (Kanabus et al., 2002). Nevertheless, bindings did not include representation of this temporal order information.

Our results fit on a larger scale with theories on action control that argue for an effect-based approach to action. These assume that actions are represented by the sensorymotor effects they produce - in fact, motor programs might be actually chosen by matching their anticipated effects against the intended effects (Hommel \& Wiers, 2017). Thus, the seemingly "natural sequence" from $\mathrm{S}$ to $\mathrm{R}$ is actually turned around from R to S (Shin, Proctor, \& Capaldi, 2010). By this reasoning, temporal order information is not as crucial as common sense might suggest.

Our results also impact on the debate concerning whether binding effects are actually related (or even identical) to learning effects. Many authors hint that binding might be an early process in learning (e.g., Dutzi \& Hommel, 2009; Frings \& Rothermund, 2011; Giesen \& Rothermund, 2014; Hommel, 1998; Moeller \& Frings, 2014), or treat binding entirely as learning, using both terms interchangeably (e.g., Henson, 2003; Horner \& Henson, 2009, 2011; Waszak, Hommel, \& Allport, 2003). However, there are also arguments and evidence for separate processes in binding and learning (e.g., Colzato, Raffone, \& Hommel, 2006; Herwig \& Waszak, 2012; Hommel \& Colzato, 2009; Moeller \& Frings, 2017). In a nutshell, it is still unclear whether binding and learning processes can be separated. The present results add some insight to this discussion. In particular, binary bindings can be interpreted to argue against identical processes in binding and (at least sequence) learning. In the serial-reaction-time (SRT) task (Nissen \& Bullemer, 1987) participants respond to serially presented stimuli, oftentimes by pressing according keys on a keyboard. Learning of a repeated stimulus and/or response sequence is indicated by a more rapid decrease of response times for structured than for unstructured sequences (Koch \& Hoffmann, 2000). To represent a sequence of several responses, obviously more than binary associations is necessary. Some sort of concatenation of the observed binary associations-where response $\mathrm{n}$ is integrated with response $n-1$ in one, and with response $n+1$ in another event file-is not possible according to the TEC, because every feature can only be integrated in one event file at the time. If learning in SRT tasks relied on bindings, either several responses (i.e., the entire sequence) would need to be represented within the same event file, or individual responses of a longer sequence would need to be integrated in 
separate event files. In the former case, order information would be missing, and in the latter binding would not be able to account for learned associations. Hence, additional mechanisms are necessary for sequence learning to emerge.

To conclude, the present results provide unambiguous evidence that bindings do not include representations of the involved elements' order, even if a sequence was clearly experienced. This is in line with theoretical accounts of SR binding. It also provides new insight into feature code representation in action control and suggests that learning cannot solely evolve from binding processes.

Author note The research reported in this article was supported by a grant of the Deutsche Forschungsgemeinschaft to Birte Moeller (MO 2839/2-1).

\section{References}

Baddeley, A. D. (1966). Short-term memory for word sequences as a function of acoustic, semantic and formal similarity. The Quarterly Journal of Experimental Psychology, 18(4), 362-365.

Colzato, L. S., Raffone, A., \& Hommel, B. (2006). What do we learn from binding features? Evidence for multilevel feature integration. Journal of Experimental Psychology: Human Perception and Performance, 32, 705-716.

Dutzi, I. B., \& Hommel, B. (2009). The microgenesis of action-effect binding. Psychological Research, 73, 425-435.

Faul, F., Erdfelder, E., Lang, A. G., \& Buchner, A. (2007). G* Power 3: A flexible statistical power analysis program for the social, behavioral, and biomedical sciences. Behavior Research Methods, 39(2), 175191.

Frings, C., \& Rothermund, K. (2011). To be or not to be...included in an event file: Integration and retrieval of distractors in stimulusresponse episodes is influenced by perceptual grouping. Journal of Experimental Psychology: Learning, Memory, and Cognition, 37, 1209-1227.

Frings, C., Schneider, K. K., \& Fox, E. (2015). The negative priming paradigm: An update and implications for selective attention. Psychonomic Bulletin \& Review, 22(6), 1577-1597.

Giesen, C., \& Rothermund, K. (2011). Affective matching moderates S-R binding. Cognition and Emotion, 25, 342-350.

Giesen, C., \& Rothermund, K. (2014). Distractor repetitions retrieve previous responses and previous targets: Experimental dissociations of distractor-response and distractor-target bindings. Journal of Experimental Psychology: Learning, Memory, and Cognition, 40, 645-659.

Gratton, G., Coles, M. G., \& Donchin, E. (1992). Optimizing the use of information: Strategic control of activation of responses. Journal of Experimental Psychology: General, 121(4), 480-506.

Henson, R. N. (2003). Neuroimaging studies of priming. Progress in Neurobiology, 70, 53-81.

Henson, R. N., Eckstein, D., Waszak, F., Frings, C., \& Horner, A. J. (2014). Stimulus-response bindings in priming. Trends in Cognitive Sciences, 18, 376-384.

Henson, R. N., Norris, D. G., Page, M. P. A., \& Baddeley, A. D. (1996). Unchained memory: Error patterns rule out chaining models of immediate serial recall. The Quarterly Journal of Experimental Psychology, 49A(1), 80-115.
Herwig, A, \& Waszak, F. (2012). Action-effect bindings and ideomotor learning in intention- and stimulus-based actions. Frontiers in Psychology, 3(444).

Hommel, B. (1998). Event files: Evidence for automatic integration of stimulus-response episodes. Visual Cognition, 5, 183-216.

Hommel, B. (2004). Event files: Feature binding in and across perception and action. Trends in Cognitive Sciences, 8, 494-500.

Hommel, B. (2005). How much attention does an event file need? Journal of Experimental Psychology: Human Perception and Performance, 31, 1067-1082.

Hommel, B. (2009). Action control according to TEC (theory of event coding). Psychological Research, 73(4), 512-526.

Hommel, B., \& Colzato, L. S. (2009). When an object is more than a binding of its features: Evidence for two mechanisms of visual feature integration. Visual Cognition, 17, 120-140.

Hommel, B., Müsseler, J., Aschersleben, G., \& Prinz, W. (2001). Codes and their vicissitudes. Behavioral and Brain Sciences, 24, 910-926.

Hommel, B., \& Wiers, R.W. (2017). Towards a unitary approach to human action control. Trends in Cognitive Sciences, 21, 940-949.

Horner, A. J., \& Henson, R. (2011). Stimulus-response bindings code both abstract and specific representations of stimuli: Evidence from a classification priming design that reverses multiple levels of response representation. Memory \& Cognition, 39, 1457-1471.

Horner, A. J., \& Henson, R. N. (2009). Bindings between stimuli and multiple response codes dominate long-lag repetition priming in speeded classification tasks. Journal of Experimental Psychology: Learning, Memory, and Cognition, 35, 757-779.

Janczyk, M., Heinemann, A., \& Pfister, R. (2012). Instant attraction: Immediate action-effect bindings occur for both, stimulus-and goal-driven actions. Frontiers in Psychology, 3, 446.

Kanabus, M., Szelag, E., Rojek, E., \& Pöppel, E. (2002). Temporal order judgement for auditory and visual stimuli. Acta Neurobiologiae Experimentalis, 62(4), 263-270.

Kiesel, A., Steinhauser, M., Wendt, M., Falkenstein, M., Jost, K., Philipp, A. M., \& Koch, I. (2010). Control and interference in task switching-A review. Psychological Bulletin, 136(5), 849.

Koch, I., \& Hoffmann, J. (2000). Patterns, chunks, and hierarchies in serial reaction-time tasks. Psychological Research, 63(1), 22-35.

Kunde, W., Kiesel, A., \& Hoffmann, J. (2003). Conscious control over the content of unconscious cognition. Cognition, 88(2), 223-242.

Logan, G. D. (1988). Toward an instance theory of automatization. Psychological Review, 95, 492-527.

Logan, G. D. (1990). Repetition priming and automaticity: Common underlying mechanisms? Cognitive Psychology, 22, 1-35.

Mayr, S., \& Buchner, A. (2006). Evidence for episodic retrieval of inadequate prime responses in auditory negative priming. Journal of Experimental Psychology: Human Perception and Performance, 32, 932-943.

Moeller, B., \& Frings, C. (2014). Attention meets binding: Only attended distractors are used for the retrieval of event files. Attention, Perception, \& Psychophysics, 76, 959-978.

Moeller, B., \& Frings, C. (2017). Dissociation of binding and learning processes. Attention, Perception, \& Psychophysics, 79(8), 25902605.

Moeller, B., Frings, C., \& Pfister, R. (2016). The structure of distractorresponse bindings: Conditions for configural and elemental integration. Journal of Experimental Psychology: Human Perception and Performance, 42, 464-479.

Moeller, B., Pfister, R., Kunde, W., \& Frings, C. (2016). A common mechanism behind distractor-response and response-effect binding?. Attention, Perception, \& Psychophysics, 78(4), 1074-1086.

Müsseler, J., \& Hommel, B. (1997). Blindness to response-compatible stimuli. Journal of Experimental Psychology: Human Perception and Performance, 23(3), 861-871. 
Nissen, M. J., \& Bullemer, P. (1987). Attentional requirements of learning: Evidence from performance measures. Cognitive Psychology, 19(1), 1-32.

Schmidt, J. R., De Houwer, J., \& Rothermund, K. (2016). The parallel episodic processing (PEP) model 2.0: A single computational model of stimulus-response binding, contingency learning, power curves, and mixing costs. Cognitive Psychology, 91, 82-108.

Shin, Y. K., Proctor, R. W., \& Capaldi, E. J. (2010). A review of contemporary ideomotor theory. Psychological Bulletin, 136(6), 943-974.
Tukey, J. (1977). Exploratory data analysis. Reading, MA: AddisonWesley.

Wagenmakers, E. J., Love, J., Marsman, M., Jamil, T., Ly, A., Verhagen, J., ... Meerhoff, F. (2017). Bayesian inference for psychology. Part II: Example applications with JASP. Psychonomic Bulletin \& Review, 25(1), 1-19.

Waszak, F., Hommel, B., \& Allport, A. (2003). Task-switching and longterm priming: Role of episodic S-R bindings in task-shift costs. Cognitive Psychology, 46, 361-413. 